\title{
Nonequilibrium spin crossover in copper phthalocyanine
}

\author{
Benjamin Siegert, ${ }^{*}$ Andrea Donarini, and Milena Grifoni \\ Institut für Theoretische Physik, Universität Regensburg, D-93040 Regensburg, Germany \\ (Received 21 July 2015; revised manuscript received 10 February 2016; published 16 March 2016)
}

\begin{abstract}
We demonstrate the nonequilibrium tip induced control of the spin state of copper phthalocyanine on an insulator coated substrate. We find that, under the condition of energetic proximity of many-body neutral excited states to the anionic ground state, the system can undergo a population inversion towards these excited states. The resulting state of the system is accompanied by a change in the total spin quantum number. Experimental signatures of the crossover are the appearance of additional nodal planes in the topographical scanning tunneling microscopy images as well as a strong suppression of the current near the center of the molecule. The robustness of the effect against moderate charge conserving relaxation processes has also been tested.
\end{abstract}

DOI: 10.1103/PhysRevB.93.121406

Introduction. Research on single molecule junctions has witnessed in recent years a broad interdisciplinary interest [1]. For example, spin dependent transport [2,3] or nuclear spin resonance [4] have been investigated. In this emergent field of molecular spintronics, spin-crossover metalorganic compounds play a prominent role [5-9]. These molecules undergo a transition between metastable spin states under the influence of external stimuli [10]. The many-body exchange interaction of the $d$ electrons on the metal center, in combination with the crystal field generated by the surrounding ligand, determines their spin state. The latter is, in three-terminal devices, governed by the same gate electrode used to tune the charge on the molecule [5-7]. Metalorganic molecules and, in particular, transition-metal phthalocyanines have also come in the focus of scanning tunneling microscopy (STM) experiments [11-18], where the charge or spin state of the molecule crucially depends on the properties of the substrate. More generally, the role itself of many-body effects in STM single molecule junctions is receiving increasing attention, both experimentally $[8,9,11-14,16,17]$ and theoretically [19-22].

In this work we demonstrate the tuning of a low-spin to high-spin transition in copper phthalocyanine $(\mathrm{CuPc})$ on an insulator coated substrate under nonequilibrium conditions. The transition can be triggered by varying the tip position or the bias voltage across the junction. The main requirements for this genuine many-body effect are an asymmetry between tip and substrate tunneling rates, which is naturally inherent to STM setups, and an energetic proximity of an excited neutral state of the molecule to its anionic ground state. As discussed below, the experimental setup is similar to that of Ref. [14], but with a slightly larger work function for the substrate. Control over the work function can be achieved by choosing different materials or crystallographic orientation for the substrate, with effects analogous to a discrete gating of the molecule. Several approaches to gate an STM junction have been very recently investigated [23-25].

Many-body Hamiltonian and spectrum of $\mathrm{CuPc}$. To properly describe the many-body electronic structure of $\mathrm{CuPc}$ is by itself a nontrivial task, since the relatively large size of

*benjamin.siegert@ur.de the molecule makes it impossible to diagonalize exactly a many-body Hamiltonian written in a local atomic basis as done for smaller molecules [26-28]. STM transport experiments on single molecules, however, are restricted to an energy window involving only the low-lying states of the molecule in its neutral, cationic and anionic configuration, with the equilibrium configuration at zero bias set by the work function $\phi_{0}$ of the substrate [14]. This allows one to use a restricted basis of frontier orbitals to construct the many-body Hamiltonian $[29,30]$. For example, for a copper substrate as in [14] is $\phi_{0}=$ $4.65 \mathrm{eV}$, and, at equilibrium, CuPc is in its neutral ground state. Thus, in the following we only retain four frontier orbitals of CuPc: the singly occupied molecular orbital (SOMO, $S$ ), the highest occupied molecular orbital (HOMO, $H$ ), and the two degenerate lowest unoccupied molecular orbitals (LUMOs, $L^{ \pm}$) [see Fig. 1(a)]. At equilibrium, the molecule contains $N_{0}=3$ frontier electrons. In this basis, all matrix elements of the Coulomb interaction are retained. Hence, besides Hubbard-like density-density interaction terms, our model also includes exchange and pair hopping terms, which ultimately are important for the structure and spin configuration of the molecular excited states.

The Hamiltonian of $\mathrm{CuPc}$ in the basis of the four single particle frontier orbitals reads

$$
\hat{\mathrm{H}}_{\mathrm{mol}}=\sum_{i} \tilde{\epsilon}_{i} \hat{n}_{i}+\frac{1}{2} \sum_{i j k l} \sum_{\sigma \sigma^{\prime}} V_{i j k l} \hat{d}_{i \sigma}^{\dagger} \hat{d}_{k \sigma^{\prime}}^{\dagger} \hat{d}_{l \sigma^{\prime}} \hat{d}_{j \sigma},
$$

where $i, j, k, l=S, H, L \pm$ and $\sigma$ is the spin degree of freedom. The energies $\tilde{\epsilon}_{i}=\epsilon_{i}+\Delta_{i}$ contain the orbital energies $\epsilon_{i}$ obtained from diagonalizing the single particle Hamiltonian $\hat{\mathrm{H}}_{0}$ of $\mathrm{CuPc}, \epsilon_{S}=-12.0 \mathrm{eV}, \epsilon_{H}=-11.7 \mathrm{eV}$, and $\epsilon_{L \pm}=-10.7 \mathrm{eV}$. The parameters $\Delta_{i}$ account for crystal field corrections and the ionic background of the molecule, since the atomic on-site energies in $\hat{\mathrm{H}}_{0}$ stem from HartreeFock calculations for isolated atoms [31]. The $\Delta_{i}$ are free parameters of the theory. Isolated CuPc has $D_{4 h}$ symmetry; the four molecular orbitals $|i \sigma\rangle$ that make up the basis of Eq. (1) transform like its $b_{1 g}(S), a_{1 u}(H)$, and $e_{g}\left(L^{ \pm}\right)$ representations. As a consequence, they acquire distinct phases $\phi_{i}$ when rotated by $90^{\circ}$ around the main symmetry axis of the molecule, as illustrated in Fig. 1(a). This yields an easy rule for the Coulomb matrix elements $V_{i j k l}$ in Eq. (1): $V_{i j k l} \neq 0$ if $\phi_{i}-\phi_{j}+\phi_{k}-\phi_{l}=0 \bmod 2 \pi$, i.e., nonvanishing 
(a)

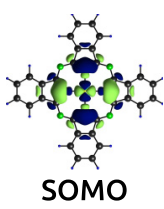

(b)



(d)

\begin{tabular}{|c|c|c|c|c|c|c|c|c|}
\hline \multicolumn{2}{|l|}{ 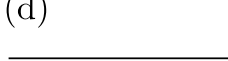 } & \multicolumn{3}{|c|}{ cation } & \multicolumn{2}{|c|}{ neutral } & \multicolumn{2}{|c|}{ anion } \\
\hline \multicolumn{9}{|c|}{ She LUMO } \\
\hline 8 & HOMO & & & & 1 & & & \\
\hline 6 & SOMO & 1 & 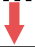 & & 1 & 1 & 1 & \\
\hline \multicolumn{2}{|c|}{$E_{N m}-E_{N 0}(\mathrm{meV})$} & 0 & 4 & 40 & 0 & $794 \quad 860$ & 18 & 374 \\
\hline \multicolumn{2}{|l|}{$S$} & & 0 & 0 & $1 / 2$ & $1 / 2 \quad 3 / 2$ & 1 & 1 \\
\hline \multicolumn{2}{|c|}{ degeneracy } & & 1 & & & 8 & 6 & 3 \\
\hline
\end{tabular}

FIG. 1. (a) Frontier orbitals used for the many-body calculation, in their complex representation. The color code shows the phase of the wave functions. (b), (c) Full and low-energy cutout, respectively, of the many-body spectrum of $\mathrm{CuPc}$ at chemical potential $\mu=$ $-4.65 \mathrm{eV}$. (d) Schematics of the lowest-lying many-body states.

contributions are only possible if the phases of the corresponding molecular orbitals add up to multiples of $2 \pi$ (quasiangular momentum conservation). These considerations remain true in the presence of a homogeneous substrate which reduces the symmetry to $C_{4 v}$. For a detailed discussion concerning the parametrization of Eq. (1) we refer to the Supplemental Material [32]. Exact numerical diagonalization of $\hat{\mathrm{H}}_{\mathrm{mol}}$ finally yields the many-body eigenenergies $E_{N m}$ and eigenstates $|N m\rangle$ of the molecule, labeled after particle number $N$ and state index $m$.

Since the molecule is in contact with the substrate and is able to exchange electrons, it is necessary to consider a grandcanonical ensemble $\hat{\mathrm{H}}_{\text {mol }}-\mu \hat{N}$, where $\mu=-\phi_{0}$. Moreover, the presence of the leads renormalizes the Hamiltonian $\hat{\mathrm{H}}_{0}$ due to image charges effects [28,33]. We model them with an effective Hamiltonian $\hat{\mathrm{H}}_{\text {mol-env }}=-\delta_{\text {ic }}\left(\hat{N}-N_{0}\right)^{2}$, being $\hat{N}$ the particle number operator on the system and $\delta_{\text {ic }}$ obtained from electrostatic considerations (see Supplemental Material). Our spectrum is fitted to the experiment of Swart et al. [14], which was conducted with $\mathrm{CuPc}$ on $\mathrm{NaCl}(3 \mathrm{ML}) / \mathrm{Cu}(100)$ $\left(\phi_{0}=4.65 \mathrm{eV}\right)$ in order to avoid polaronic charge bistability, by using a constant shift $\Delta_{i}=\Delta=1.83 \mathrm{eV}$, a dielectric constant $\epsilon_{\mathrm{mol}}=2.2$ in the evaluation of the matrix elements $V_{i j k l}$, and an image-charge renormalization $\delta_{\text {ic }}=0.32 \mathrm{eV}$.

Figures 1(b) and 1(c) show the cationic, neutral, and anionic subblocks of the many particle spectrum and their degeneracies. A schematic depiction of these states is shown in Fig. 1(d). The actual states are linear combinations of several Slater determinants. Only the dominant contribution is shown and spin multiplets are represented by the configuration of highest $S_{z}$. The neutral ground state has a doublet structure (total spin $S=\frac{1}{2}$ ) arising from the doubly filled HOMO and the unpaired spin in the SOMO. The cationic and anionic ground states are triplets $(S=1)$. The former has a singly filled HOMO; the latter a singly filled LUMO. Both form spin triplets (and singlets, $S=0$, for the first excited states) with the singly filled SOMO. We find a triplet anionic ground state with a triplet-singlet splitting of $18 \mathrm{meV}$, in agreement with independent experimental measurements [11] (measured splitting of $21 \mathrm{meV}$ ). The first and second excited states of the neutral molecule are found to be a doublet $\left(S=\frac{1}{2}\right)$ and a quadruplet $\left(S=\frac{3}{2}\right)$, respectively, with additional twofold orbital degeneracy.

Transport dynamics and spin crossover. The full system is characterized by the Hamiltonian $\hat{\mathrm{H}}=\hat{\mathrm{H}}_{\text {mol }}+\hat{\mathrm{H}}_{\text {mol-env }}+$ $\hat{\mathrm{H}}_{\mathrm{S}}+\hat{\mathrm{H}}_{\mathrm{T}}+\hat{\mathrm{H}}_{\text {tun }}$, where $\hat{\mathrm{H}}_{\mathrm{S}}$ and $\hat{\mathrm{H}}_{\mathrm{T}}$ describe noninteracting electronic reservoirs for substrate (S) and tip (T). The tunneling Hamiltonian is $\hat{\mathrm{H}}_{\text {tun }}=\sum_{\eta \mathbf{k} i \sigma} t_{\mathbf{k} i}^{\eta} \hat{c}_{\eta \mathbf{k} \sigma}^{\dagger} \hat{d}_{i \sigma}+$ H.c., where $\hat{c}_{\eta \mathbf{k} \sigma}^{\dagger}$ creates an electron in lead $\eta$ with spin $\sigma$ and momentum $\mathbf{k}$. The tunneling matrix elements $t_{\mathbf{k} i}^{\eta}$ are obtained analogously to Ref. [19]. The dynamics is calculated via a generalized master equation for the reduced density operator $\rho_{\text {red }}=\operatorname{Tr}_{\mathrm{S}, \mathrm{T}}(\rho)$ (see Refs. [19,27]). In particular, we focus on $\rho_{\text {red }}^{\infty}$, solving the stationary equation $\mathcal{L}\left[\rho_{\text {red }}\right]=0$, where $\mathcal{L}$ is the Liouvillian superoperator.

In analogy to Ref. [34], we include a phenomenological relaxation term $\mathcal{L}_{\text {rel }}$ in the Liouvillian [35]:

$$
\mathcal{L}_{\text {rel }}[\rho]=-\frac{1}{\tau}\left(\rho-\sum_{N m} \rho_{m m}^{\mathrm{th}, N}|N m\rangle\langle N m| \sum_{n} \rho_{n n}^{N}\right) .
$$

It is proportional to the deviation of the reduced density matrix from the thermal one $\rho^{\text {th }}$, which is given by the Boltzmann distribution $\rho_{m m}^{\mathrm{th}, N} \sim \exp \left(-\frac{E_{N m}}{k_{B} T}\right)$ with $\sum_{m} \rho_{m m}^{\mathrm{th}, N}=1$. Since $\mathcal{L}_{\text {rel }}$ describes relaxation processes which conserve the particle number on the molecule, it does not contribute directly to the current. The relaxation rate $\frac{1}{\tau}$ is taken of the same order of magnitude as the tip tunneling rate. The Liouvillian $\mathcal{L}=\mathcal{L}_{\text {rel }}+\sum_{\eta} \mathcal{L}_{\eta}$ decomposes into the relaxation term and sub-Liouvillians for each lead. Sorting of the occurring terms after substrate and tip contributions yields the current operator of the respective lead $\eta$ as $\hat{I}_{\eta}=\hat{N} \mathcal{L}_{\eta}$, from which the stationary current through the system is evaluated.

Results of our transport calculations are presented in Fig. 2. In panels (a), (d), and (g) we show constant height current maps, constant current topographies in (b), (e), and (h), and in (c), (f), and (i) the expectation value of the total spin of the molecule depending on the tip position, $S_{\mathbf{r}_{\mathrm{T}}}=\sqrt{\left\langle\hat{S}^{2}\right\rangle_{\mathbf{r}_{\mathrm{T}}}+\frac{1}{4}}-$ $\frac{1}{2}$ where $\left\langle\hat{S}^{2}\right\rangle_{\mathbf{r}_{\mathrm{T}}}=\operatorname{Tr}_{\text {mol }}\left[\hat{S}^{2} \rho_{\text {red }}^{\infty}\left(\mathbf{r}_{\mathrm{T}}\right)\right]$. The upper three panels (a), (b), and (c) are for a work function of $\phi_{0}=4.65 \mathrm{eV}$ (standard case) and a bias voltage of $V_{\mathrm{b}}=-2.72 \mathrm{~V}$. At this bias the cationic resonance is occurring. Since the neutral and cationic ground states only differ in the occupation of the HOMO [see Fig. 1(d)], tunneling occurs via this orbital and the maps (a) and (b) resemble its structure. With the same work function $\phi_{0}=4.65 \mathrm{eV}$, the anionic resonance is taking 

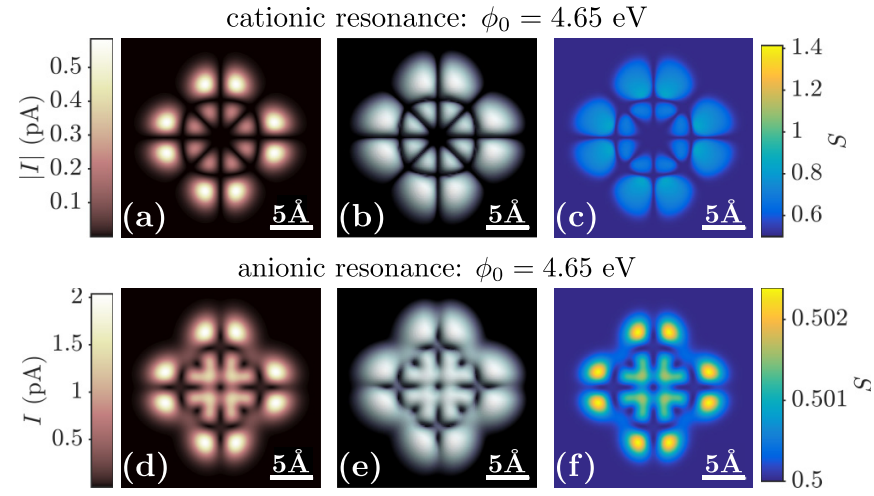

anionic resonance: $\phi_{0}=5 \mathrm{eV}$

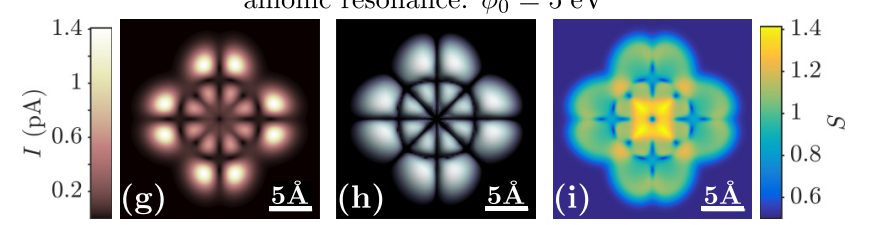

FIG. 2. Constant height current maps [(a),(d),(g)], constant current topographies [(b),(e),(h)], and maps of the system's total spin $S$ [(c),(f),(i)]. Constant height and spin maps are calculated for a tip-molecule distance of $5 \AA$, and constant current topographies for currents $I=0.5,0.75$, and $1.0 \mathrm{pA}$ for panels (b), (e), and (h), respectively.

place at the positive bias $V_{\mathrm{b}}=0.81 \mathrm{~V}$ [see Figs. 2(d) and 2(e)]. For equivalent reasons as for the former case, tunneling is happening via the LUMO and the spatial dependence of the maps resembles the topography of this orbital. Panels $(\mathrm{g}),(\mathrm{h})$, and (i) are instead calculated at $\phi_{0}=5 \mathrm{eV}$ (anomalous case), again at the anionic resonance, now shifted to $V_{\mathrm{b}}=1.38 \mathrm{~V}$ due to the larger work function. Notice that the moderate increase of the latter does not imply any charging or orbital reordering. Thus, panels (g) and (h) are puzzling. Despite referring to an anionic resonance, they closely resemble the HOMO [cf. panels (a) and (b)]. This anomalous topography cannot be explained by standard single orbital tunneling.

Panels (c), (f), and (i) reveal the tip-position dependent expectation value of the molecular spin. At the standard anionic transition, panel (f), the spin remains essentially constant. At the standard cationic transition, panel (c), the rather homogeneous enhancement of the molecular spin is due to small populations of a large number of excited states, made accessible by the large resonance bias $\left(V_{\text {res }}=-2.7 \mathrm{~V}\right)$. It is the anomalous anionic transition, panel (i), to show the largest variation of the molecular spin, concentrated at the positions of the anomalous current suppression [compare panels (g) and (d)]. To explain the unconventional properties shown in Fig. 2, we examine bias traces calculated for different tip positions and values of the work function. Figure 3(a) shows a shift of the anionic resonant peak in the $\frac{d I}{d V}$ for the anomalous case. The value $V_{\text {res }}$ at which the peak is expected is given by

$$
V_{\text {res }}\left(\phi_{0}\right)=\frac{1}{\alpha_{\mathrm{T}}|e|}\left(E_{N_{0}+1,0}-E_{N_{0}, 0}-\delta_{\text {ic }}+\phi_{0}\right),
$$

where $\alpha_{\mathrm{T}}$ is the fraction of bias drop between tip and molecule, and $E_{N, 0}$ is the energy of the $N$-particle ground state. The shift of the resonance to lower biases seen in Fig. 3(a) suggests the

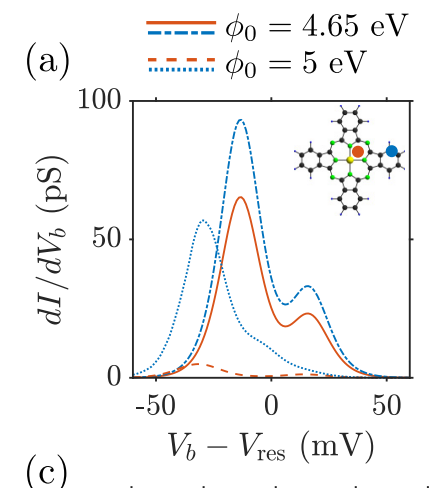

(b)


FIG. 3. (a) Differential conductance and (b) total spin curves taken at different tip positions and work functions around the bias $V_{\text {res }}\left(\phi_{0}\right)$ of the anionic resonance. The inset in (b) shows the change of the spin for the standard case in magnification. (c) Populations of the density matrix around $V_{\text {res }}\left(\phi_{0}\right)$. Left panel: standard case, $\phi_{0}=4.65 \mathrm{eV}$. Middle (right) panel: anomalous case, $\phi_{0}=5 \mathrm{eV}$, with tip near the center (outer on the ligand).

appearance of a population inversion from the neutral ground state to an excited state. The transitions from the latter to the anionic ground state open in fact at much lower biases. Also, the evolution of the spin of the molecule shown in Fig. 3(b) reinforces this proposition. In the anomalous case, the change of the system from a low to a high spin state, as well as the saturation of the spin, can be clearly seen. This contrasts the normal anionic transition, where only a marginal change is observable. In Fig. 3(c) we show the evolution of the eigenvalues of the stationary density matrix $\rho_{\text {red }}^{\infty}$, i.e., the populations of the physical basis [27], around the anionic resonance $V_{\text {res }}\left(\phi_{0}\right)$, depending on work function and tip position. In the standard case [left panel of Fig. 3(c)], the system is effectively always in its neutral ground state, independently of the tip position. For the anomalous case (middle and right panels of Fig. 3) there is a remarkable depopulation of the neutral ground state in favor of different excited states, depending on the position of the tip. Moreover, no charging is detectable.

We focus now on the mechanism yielding the population inversion with associated spin crossover. In the standard case a sufficiently high bias opens the transition from the neutral to the anionic ground state, with an electron tunneling into the LUMO. By consecutive tunneling to the substrate, the system quickly returns into its neutral ground state [see Fig. 4(a)]. The strong asymmetry between the tip and substrate rates keeps the system essentially in its neutral ground state $\left(S=\frac{1}{2}\right)$.

Due to the proximity of the many-body eigenenergies, in the anomalous case, new transport channels are opening. 
(a) standard

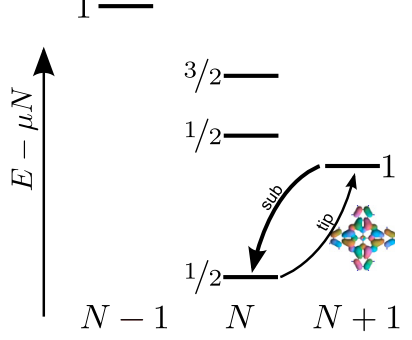

(c)



(b)

anomalous
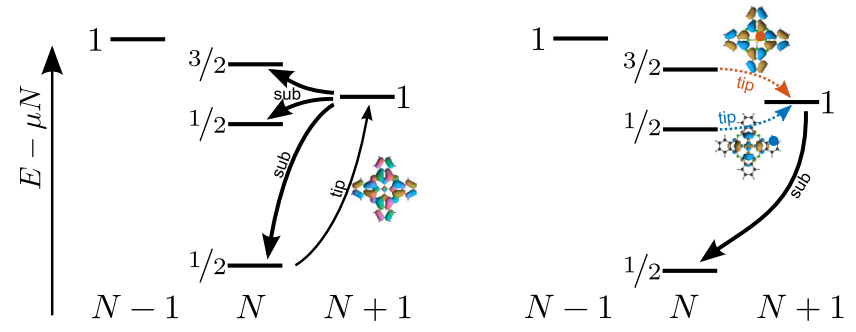

FIG. 4. (a) and (b) Sketches of the tunneling processes at the anionic resonance for the standard $\left(\phi_{0}=4.65 \mathrm{eV}\right)$ and the anomalous $\left(\phi_{0}=5 \mathrm{eV}\right)$ case, respectively. (c) Phase diagram defining the class of anomalous junctions. The dashed line represents $\mathrm{CuPc}$, with the dots referring to the work functions considered in this Rapid Communication.

The system acquires a finite probability to leave the anionic ground state and reach a neutral excited state by releasing an electron to the substrate. The molecule can only return to its neutral ground state either via charge conserving relaxation or by successive transitions to the anionic ground state via the tip, and from there to the neutral ground state via the substrate. However, the tip tunneling event acts as a bottleneck and depends on the tip position [cf. right panel in Fig. 4(b)]. Leaving the first excited state $\left(S=\frac{1}{2}\right)$ requires tunneling into the SOMO, while leaving the second excited state $\left(S=\frac{3}{2}\right)$ would require tunneling into the HOMO. Additionally, near the center of the molecule the HOMO is vanishing, whereas on the outer ligand part the SOMO has little to no amplitude. Therefore, tunneling into these orbitals at the respective positions is strongly suppressed and the system ultimately ends up in the corresponding neutral excited states.

The dynamics just described is not a peculiarity of $\mathrm{CuPc}$. As detailed in the Supplemental Material, the nonequilibrium spin crossover and the corresponding anomalous topography should be observable in an entire class of molecular junctions [see Fig. 4(c)]. The most important condition defining this class is given by $\Delta_{\text {tr }}>2 \Delta_{\text {opt }}$, being $\Delta_{\text {tr }}=$ IP $-\mathrm{EA}-2 \delta_{\text {ic }}$ the transport gap and $\Delta_{\text {opt }}=E_{N_{0}, 1}-E_{N_{0}, 0}$ the optical gap, with IP and EA the ionization potential and the electron affinity, respectively. Additionally, the range of appropriate work functions should be found in the vicinity of $\phi_{\text {sym }}=$ $(\mathrm{IP}+\mathrm{EA}) / 2$.

Conclusions. For an experimentally accessible substrate work function $\phi_{0}=5 \mathrm{eV}$, we predict the appearance, in proximity to the anionic resonance, of a population inversion between the neutral ground and excited states of $\mathrm{CuPc}$. Depending on the tip position, the molecule is triggered into a low-spin $\left(S=\frac{1}{2}\right)$ to high-spin $\left(S=\frac{3}{2}\right)$ transition which is mediated by this population inversion. The latter is experimentally observable via dramatic changes in the topographical properties of constant height and constant current STM images, compared to a standard LUMO-mediated anionic transition. Direct observation of the spin crossover might be accessible using spin-polarized scanning probe microscopy techniques [36]. The effect is also robust against moderate charge conserving relaxation processes and should be observable in a rather wide class of molecular junctions.

Acknowledgments. The authors thank Richard Korytar, Thomas Niehaus, Jascha Repp, and Dmitry Ryndyk for fruitful discussions. Funding by the Deutsche Forschungsgemeinschaft within the research program SFB 689 is acknowledged.
[1] S. V. Aradhya and L. Venkataraman, Nat. Nanotechnol. 8, 399 (2013).

[2] L. Bogani and W. Wernsdorfer, Nat. Mater. 7, 179 (2008).

[3] S. Sanvito, Chem. Soc. Rev. 40, 3336 (2011).

[4] S. Thiele, F. Balestro, R. Ballou, S. Klyatskaya, M. Ruben, and W. Wernsdorfer, Science 344, 1135 (2014).

[5] A. S. Zyazin, J. W. G. van den Berg, E. A. Osorio, H. S. J. van der Zant, N. P. Konstantinidis, M. Leijnse, M. R. Wegewijs, F. May, W. Hofstetter, C. Danieli, and A. Cornia, Nano Lett. 10, 3307 (2010).

[6] E. A. Osorio, K. Moth-Poulsen, H. S. J. van der Zant, J. Paaske, P. Hedegard, K. Flensberg, J. Bendix, and T. Bjornholm, Nano Lett. 10, 105 (2010).

[7] V. Meded, A. Bagrets, K. Fink, R. Chandrasekar, M. Ruben, F. Evers, A. Bernand-Mantel, J. S. Seldenthuis, A. Beukman, and H. S. J. van der Zant, Phys. Rev. B 83, 245415 (2011).
[8] T. Miyamachi, M. Gruber, V. Davesne, M. Bowen, S. Boukari, L. Joly, F. Scheurer, G. Rogez, T. K. Yamada, P. Ohresser, E. Beaurepaire, and W. Wulfhekel, Nat. Commun. 3, 938 (2012).

[9] T. G. Gopakumar, F. Matino, H. Naggert, A. Bannwarth, F. Tuczek, and R. Berndt, Angew. Chem., Int. Ed. 51, 6262 (2012).

[10] P. Gütlich, Y. Garcia, and H. A. Goodwin, Chem. Soc. Rev. 29, 419 (2000).

[11] A. Mugarza, C. Krull, R. Robles, S. Stepanow, G. Ceballos, and P. Gambardella, Nat. Commun. 2, 490 (2011).

[12] A. Mugarza, R. Robles, C. Krull, R. Korytár, N. Lorente, and P. Gambardella, Phys. Rev. B 85, 155437 (2012).

[13] B. W. Heinrich, L. Braun, J. I. Pascual, and K. J. Franke, Nat Phys. 9, 765 (2013).

[14] I. Swart, T. Sonnleitner, and J. Repp, Nano Lett. 11, 1580 (2011).

[15] C. Uhlmann, I. Swart, and J. Repp, Nano Lett. 13, 777 (2013).

[16] L. Liu, T. Dienel, R. Widmer, and O. Gröning, ACS Nano 9, 10125 (2015). 
[17] F. Schulz, M. Ijäs, R. Drost, S. K. Hämäläinen, A. Harju, A. P. Seitsonen, and P. Liljeroth, Nat. Phys. 11, 229 (2015).

[18] B. W. Heinrich, L. Braun, J. I. Pascual, and K. J. Franke, Nano Lett. 15, 4024 (2015)

[19] S. Sobczyk, A. Donarini, and M. Grifoni, Phys. Rev. B 85, 205408 (2012).

[20] D. Toroz, M. Rontani, and S. Corni, J. Chem. Phys. 134, 024104 (2011).

[21] A. Donarini, B. Siegert, S. Sobczyk, and M. Grifoni, Phys. Rev. B 86, 155451 (2012).

[22] D. Toroz, M. Rontani, and S. Corni, Phys. Rev. Lett. 110, 018305 (2013).

[23] I. Fernández-Torrente, D. Kreikemeyer-Lorenzo, A. Stróżecka, K. J. Franke, and J. I. Pascual, Phys. Rev. Lett. 108, 036801 (2012).

[24] S. Bouvron, Gate-controlled scanning tunneling spectroscopy of CoPc molecules on graphene, Ph.D. thesis, Faculty of Physics, Universtiy of Konstanz, 2014.

[25] J. Martinez-Blanco, C. Nacci, S. C. Erwin, K. Kanisawa, E. Locane, M. Thomas, F. von Oppen, P. W. Brouwer, and S. Fölsch, Nat. Phys. 11, 640 (2015).

[26] M. H. Hettler, W. Wenzel, M. R. Wegewijs, and H. Schoeller, Phys. Rev. Lett. 90, 076805 (2003).

[27] D. Darau, G. Begemann, A. Donarini, and M. Grifoni, Phys. Rev. B 79, 235404 (2009).
[28] K. Kaasbjerg and K. Flensberg, Phys. Rev. B 84, 115457 (2011).

[29] D. A. Ryndyk, A. Donarini, M. Grifoni, and K. Richter, Phys. Rev. B 88, 085404 (2013).

[30] A. Chiesa, S. Carretta, P. Santini, G. Amoretti, and E. Pavarini, Phys. Rev. Lett. 110, 157204 (2013).

[31] J. B. Mann, Atomic Structure Calculations I. HartreeFock Energy Results for the Elements Hydrogen to Lawrencium, Technical Report No. LA-3690, Los Alamos Scientific Laboratory of the University of California, 1967.

[32] See Supplemental Material at http://link.aps.org/supplemental/ 10.1103/PhysRevB.93.121406 for further details.

[33] M. L. Perrin, C. J. O. Verzijl, C. A. Martin, A. J. Shaikh, R. Eelkema, J. H. van Esch, J. M. van Ruitenbeek, J. M. Thijssen, H. S. J. van der Zant, and D. Dulic, Nat. Nanotechnol. 8, 282 (2013).

[34] J. Koch and F. von Oppen, Phys. Rev. Lett. 94, 206804 (2005).

[35] Differently from Ref. [34] we include in (2) also coherences. $\mathcal{L}_{\text {rel }}$ accounts thus also for dephasing. For simplicity, we assume the same phenomenological rate for dephasing and dissipation.

[36] F. Pielmeier and F. J. Giessibl, Phys. Rev. Lett. 110, 266101 (2013). 